\title{
Correction to: Chromosome counts in Chaetogastra (Melastomataceae, Melastomateae)
}

Fabrício Schmitz Meyer ${ }^{1}$, Klenya Rocha Braga ${ }^{1}$, Eliana R. Forni-Martins ${ }^{2}$, and RenAto GoldenBerg ${ }^{3}$

${ }^{1}$ Programa de Pós-Graduação em Biologia Vegetal, Universidade Estadual de Campinas, CEP 13083-862, Campinas, São Paulo, Brazil; e-mail: fabriciovegetal@yahoo.com.br

${ }^{2}$ Departamento de Biologia Vegetal, Universidade Estadual de Campinas, CEP 13083-862, Campinas, São Paulo, Brazil

${ }^{3}$ Departamento de Botânica, Universidade Federal do Paraná, CEP 81531-970, Curitiba, Paraná, Brazil

\section{Correction to: Brittonia \\ https://doi.org/10.1007/s12228- \\ 018-9522-8}

This article was published with an incorrect title. The correct title is: Chromosome counts in Chaetogastra (Melastomataceae, Melastomateae). The original article has been corrected. 